\title{
The isobaric pulmonary arterial compliance in pulmonary hypertension
}

\author{
Denis Chemla ${ }^{1,2,3}$, Emmanuelle Berthelot ${ }^{2,4}$, Jason Weatherald (10 ${ }^{5}$, \\ Edmund M. T. Lau ${ }^{6}$, Laurent Savale (10 ${ }^{2,3,7}$, Antoine Beurnier ${ }^{1,2,4}$, \\ David Montani (10,3,7, Olivier Sitbon (102,3,7, Pierre Attal (10 ${ }^{1,8}$, David Boulate ${ }^{9}$, \\ Patrick Assayag ${ }^{2,3,4}$, Marc Humbert (10,3,7 and Philippe Hervé 3,9
}

\begin{abstract}
Affiliations: 'Service d'explorations fonctionnelles multidisciplinaires bi-site Antoine Béclère - Kremlin Bicêtre, GHU Paris Sud, DMU-CORREVE, AP-HP, Le Kremlin-Bicêtre, France. ${ }^{2}$ Université Paris-Sud, Faculté de Médecine, Université Paris-Saclay, Le Kremlin-Bicêtre, France. ${ }^{3}$ INSERM UMR_S 999, Hôpital Marie Lannelongue, Le Plessis Robinson, France. ${ }^{4}$ Service de cardiologie, GHU Paris Sud, AP-HP, Le KremlinBicêtre, France. ${ }^{5}$ Dept of Medicine, Division of Respirology, University of Calgary, and Libin Cardiovascular Institute, Calgary, AB, Canada. ${ }^{6}$ Dept of Respiratory Medicine, Royal Prince Alfred Hospital, University of Sydney, Camperdown, NSW, Australia. ${ }^{7}$ Service de Pneumologie, Hôpital Bicêtre, AP-HP, Le Kremlin-Bicêtre, France. ${ }^{8}$ Dept of Otolaryngology - Head and Neck Surgery, Shaare-Zedek Medical Center and Hebrew University Medical School, Jerusalem, Israel. ${ }^{9}$ Departement de Chirurgie Thoracique, Vasculaire et de Transplantation Pulmonaire, Hopital Marie Lannelongue, Le Plessis Robinson, France.
\end{abstract}

Correspondence: Denis Chemla, Service des Explorations Fonctionnelles, Broca 4, Hôpital de Bicêtre, 78 rue du Général Leclerc, 94275 Le Kremlin Bicêtre, France. E-mail: denis.chemladaphp.fr

ABSTRACT Pulmonary hypertension is associated with stiffening of pulmonary arteries which increases right ventricular pulsatile loading. High pulmonary artery wedge pressure (PAWP) in postcapillary pulmonary hypertension (Pc-PH) further decreases pulmonary arterial compliance (PAC) at a given pulmonary vascular resistance (PVR) compared with precapillary pulmonary hypertension, thus responsible for a higher total arterial load. In all other vascular beds, arterial compliance is considered as mainly determined by the distending pressure, due to non-linear stress-strain behaviour of arteries. We tested the applicability, advantages and drawbacks of two comparison methods of PAC depending on the level of mean pulmonary arterial pressure (mPAP; isobaric PAC) or PVR.

Right heart catheterisation data including PAC (stroke volume/pulse pressure) were obtained in 112 Pc$\mathrm{PH}$ (of whom 61 had combined postcapillary and precapillary pulmonary hypertension) and 719 idiopathic pulmonary arterial hypertension (iPAH).

PAC could be compared over the same mPAP range $(25-66 \mathrm{mmHg})$ in $792(95.3 \%)$ out of 831 patients and over the same PVR range (3-10.7 WU) in only $520(62.6 \%)$ out of 831 patients. The main assumption underlying comparisons at a given PVR was not verified as the PVR $\times$ PAC product (RC-time) was not constant but on the contrary more variable than mPAP. In the 788/831 (94.8\%) patients studied over the same PAC range $\left(0.62-6.5 \mathrm{~mL} \cdot \mathrm{mmHg}^{-1}\right)$, PVR and thus total arterial load tended to be higher in iPAH.

Our study favours comparing PAC at fixed mPAP level (isobaric PAC) rather than at fixed PVR. A reappraisal of the effects of PAWP on the pulsatile and total arterial load put on the right heart is needed, and this point deserves further studies.

@ERSpublications

In postcapillary and precapillary pulmonary hypertension patients, this study favours comparing pulmonary arterial compliance (PAC) at fixed mean pulmonary artery pressure level (isobaric PAC) rather than at fixed pulmonary vascular resistance level https://bit.ly/3aTLYdS

Cite this article as: Chemla D, Berthelot E, Weatherald J, et al. The isobaric pulmonary arterial compliance in pulmonary hypertension. ERJ Open Res 2021; 7: 00941-2020 [https://doi.org/10.1183/ 23120541.00941-2020]. 


\section{Introduction}

Pulmonary hypertension is a group of conditions characterised by an increased mean pulmonary artery pressure (mPAP) at rest due to increased pulmonary vascular resistance (PVR) (precapillary pulmonary hypertension), increased left atrial pressure, as reflected currently by pulmonary artery wedge pressure PAWP (postcapillary pulmonary hypertension, Pc-PH), or increased cardiac output (hyperkinetic pulmonary hypertension) [1-3]. While the abnormally high mPAP reflects the increased steady load put on the right heart, pulmonary hypertension is also associated with a stiffening of pulmonary arteries which leads to an increased pulsatile load [4-8]. Total pulmonary arterial compliance ( $\mathrm{PAC}=1 /$ pulmonary arterial stiffness) is defined as the relation between changes in arterial volume per unit change in arterial pressure, and it is currently estimated by the stroke volume over pulmonary arterial pulse pressure ratio (SV/PP) [9-11]. In patients with pulmonary hypertension, increased PVR and reduced PAC may contribute to right ventricular dysfunction and poor prognosis [12-19].

Haemodynamic studies performed nowadays consider as granted that the major determinants of PAC are PVR and PAWP. There is a curvilinear negative relationship between PVR and PAC in precapillary pulmonary hypertension patients, and this relationship is shifted downwards in Pc-PH [20-24]. In other words, the elevations in PAWP lead to lower PAC than would be anticipated from elevated PVR alone. It is thus admitted that the total arterial load is higher in postcapillary than in precapillary pulmonary hypertension $[11,20,24]$. This pathophysiological framework may be viewed as a unique feature of the pulmonary circulation. Indeed, in all other vascular beds, arterial compliance is determined by the interplay between the distending pressure and the intrinsic properties of the arterial wall, not by vascular resistance and downstream pressure $[25,26]$. The rationale for comparing PAC at a given PVR level is based on the key assumption that PAC is accurately predicted by PVR alone in either group, and this assumption relies on the constancy of the PVR times PAC product ("RC-time constancy") [20-24].

In the present study performed in precapillary and postcapillary pulmonary hypertension patients, we tested the applicability, advantages and drawbacks of two comparison methods of PAC depending on either the level of PVR or the prevailing distending pressure mPAP (isobaric PAC). We also tested the hypothesis that the combined effects of PVR and PAC result in a higher total arterial load in postcapillary compared with precapillary pulmonary hypertension patients.

\section{Methods}

\section{Patient population}

This was a retrospective comparison of right heart catheterisation results prospectively obtained 1) in Pc-PH patients referred to our French pulmonary hypertension referral centre for known or suspected pulmonary hypertension between June 2012 and July $2015(\mathrm{n}=112)$; and 2) in the previously described 719 idiopathic pulmonary arterial hypertension (iPAH) patients belonging to the French PAH Network 2006-2016 registry [7]. Only adult patients with their full haemodynamic data set available (pulmonary arterial pressures, PAWP, cardiac output and heart rate) entered the study. The final diagnosis was according to current guidelines at the time of the study, namely $\mathrm{mPAP} \geqslant 25 \mathrm{mmHg}, \mathrm{PAWP} \leqslant 15 \mathrm{mmHg}$, and $\mathrm{PVR}>3$ Wood units (WU) (iPAH) or $\mathrm{mPAP} \geqslant 25 \mathrm{mmHg}$ and $\mathrm{PAWP}>15 \mathrm{mmHg}$ (Pc-PH). Among $\mathrm{Pc}-\mathrm{PH}$ patients, the isolated $\mathrm{Pc}-\mathrm{PH}$ patients (Ipc-PH) had $\mathrm{PVR} \leqslant 3 \mathrm{WU}$ while the combined post- and pre-capillary pulmonary hypertension patients $(\mathrm{Cpc}-\mathrm{PH})$ had $\mathrm{PVR}>3 \mathrm{WU}$ [2]. Atrial fibrillation was documented in $31(28 \%)$ Pc-PH patients, and in $45(6 \%)$ iPAH $(\mathrm{p}<0.05)$. This study complied with the Declaration of Helsinki. Although French law does not require ethics committee approval or informed consent for retrospective data collection, the data collected were anonymised and complied with the requirements of the Commission Nationale Informatique et Liberté (CNIL). This organisation dedicated to privacy, information technology and civil rights in France, approved the methods used to collect and analyse data (May 24, 2003; approval number 842063).

\section{Measurements and data analysis}

Pulmonary haemodynamics were acquired with a balloon-tipped, double lumen, fluid-filled 7F Swan Ganz catheter via the jugular or brachial vein approach $[7,27]$. Zero reference was set at the mid-chest. Pressure measurements were averaged over the respiratory cycle. Cardiac output was measured by thermodilution and three values differing by $<10 \%$ were averaged. The transpulmonary pressure gradient TPG was calculated as the mPAP minus PAWP difference. The PVR was calculated as the TPG/cardiac output ratio. Indexed values of haemodynamic parameters were also calculated using correction for body surface area. The PAC was calculated as SV/PP where SV is the thermodilution stroke volume (cardiac output /heart rate) and PP is pulmonary arterial pulse pressure (systolic PAP minus diastolic PAP). The time constant of PAP decline in diastole (RC-time) was estimated using the empiric product $\mathrm{PVR} \times \mathrm{PAC}$. The isobaric $\mathrm{PAC}$ was the total pulmonary arterial stiffness at a given mPAP level. 


\section{Statistics}

Normality was assessed with the Kolmogorov-Smirnov test. Continuous variables were expressed as median (interquartile range) as the vast majority of the variables were non-normally distributed. The data dispersion was quantified using the quartile coefficient of dispersion, the interquartile ratio, and the acceptance range for $95 \%$ of the values [27, 28]. We considered a claim for the presence of a "near-constant" variable in the database to be at least reasonable if the $95 \%$ limits of agreement fall within the clinically acceptable range of up to $\pm 30 \%$, i.e., if $95 \%$ of the computed values were located within $30 \%$ of the median value [29]. The Spearman's rank correlation coefficient (rho) was used as a nonparametric measure of rank correlation. Comparisons were performed using the Mann-Whitney's test (independent samples) and Chi-squared test. All tests were two-sided with a $\mathrm{p}<0.05$ considered statistically significant. Statistical analyses were performed using the MEDCALC 8.1.0.0 software (MedCalc Software, Mariakerke, Belgium)

\section{Results}

The clinical and haemodynamic characteristics of the study population are given in tables 1 and 2. As compared to Pc-PH patients, iPAH had higher mPAP, higher PVR and lower PAC (each p<0.05). Compared with Ipc-PH patients, Cpc-PH had higher mPAP, higher PVR and lower PAC (each p<0.05). As compared to Cpc-PH, iPAH had similar mPAP ( $\mathrm{p}=0.34)$, markedly higher PVR $(+207 \%)$ and slightly lower PAC $(-23 \%)$ (each $\mathrm{p}<0.05)$. In Pc-PH, there was no relationship between RAP and PAC $(\mathrm{p}=0.47)$.

\section{Applicability of comparison methods}

When PAC values were studied over the same PVR range (3-10.7 WU), only $520(62.6 \%)$ out of 831 patients entered the comparison, namely 61 (54.5\%) out of $112 \mathrm{Pc}-\mathrm{PH}$ patients and 459 (63.8\%) out of $719 \mathrm{iPAH}$ (figure 1 left panel). By definition, the Ipc-PH (51 (45.5\%) out of 112 of Pc-PH) had their PVR $<3$ WU and thus could not be compared to iPAH at fixed PVR. Furthermore, 260 (36.2\%) out of 719 iPAH did not enter the comparison as their PVR exceeded the highest PVR values documented in $\mathrm{Cpc}-\mathrm{PH}(10.7 \mathrm{WU})$. On the other hand, when PAC values were studied over the same mPAP range $(25-66 \mathrm{mmHg}), 792(95.3 \%)$ out of 831 patients entered the comparison, namely $112 \mathrm{Pc}-\mathrm{PH}$ patients $(100 \%)$ and $680(94.6 \%)$ out of $719 \mathrm{iPAH}$ (figure 1 right panel). Only 39 (4.7\%) out of $831 \mathrm{iPAH}$ patients could not enter the comparison as their mPAP was $>66 \mathrm{mmHg}$.

TABLE 1 Demographic and haemodynamic characteristics of the patients with idiopathic pulmonary arterial hypertension (iPAH) and postcapillary pulmonary hypertension (Pc-PH)

\begin{tabular}{|c|c|c|}
\hline & iPAH & $\mathrm{Pc}-\mathrm{PH}$ \\
\hline Patients & 719 & 112 \\
\hline Age years & $66(51-75)$ & $70.5(64-79)$ \\
\hline Female sex & $386(54)$ & 72 (64) NS \\
\hline Body surface area $\mathrm{m}^{2}$ & $1.77(1.63-1.94)$ & $1.92(1.79-2.06)$ \\
\hline Cardiac output L.min ${ }^{-1}$ & $4.18(3.38-5.00)$ & $5.49(4.33-6.29)$ \\
\hline Cardiac index L.min ${ }^{-1} \cdot \mathrm{m}^{-2}$ & $2.35(2.90-2.79)$ & $2.77(2.29-3.35)$ \\
\hline Heart rate beats. $\min ^{-1}$ & $79(68-88)$ & $78(63-88)$ NS \\
\hline Stroke volume $\mathrm{mL}$ & $54(42-69)$ & 70 (55-93) \\
\hline Stroke volume index $\mathrm{mL} \cdot \mathrm{m}^{-2}$ & $30(24-38)$ & $37(29-48)$ \\
\hline mPAP $\mathrm{mmHg}$ & $46(39-54)$ & $38(33-48)$ \\
\hline Pulmonary arterial pulse pressure $\mathrm{mmHg}$ & $45(36-53)$ & $36(26-43)$ \\
\hline PAWP $\mathrm{mmHg}$ & $9(6-11)$ & $21(17-25)$ \\
\hline TPG $\mathrm{mmHg}$ & $38(31-46)$ & $18(12-25)$ \\
\hline RAP $\mathrm{mmHg}$ & $7(4-11)$ & $13(10-18)$ \\
\hline PVR WU & $9.1(6.6-12.5)$ & $3.3(2.4-4.7) \mathrm{NA}$ \\
\hline PAC $\mathrm{mL} \cdot \mathrm{mmHg}^{-1}$ & $1.20(0.88-1.68)$ & $2.18(1.46-3.07)$ \\
\hline RC-time ms & $650(546-767)$ & 396 (321-489) \\
\hline \multicolumn{3}{|c|}{$\begin{array}{l}\text { Data are presented as } n \text {, median (interquartile range) or } n(\%) \text {. mPAP: mean pulmonary artery pressure; } \\
\text { PAC: total pulmonary arterial compliance; PAH: pulmonary arterial hypertension; PAWP: pulmonary artery } \\
\text { wedge pressure; PVR: pulmonary vascular resistance; RAP: right atrial pressure; RC-time: the PVR times } \\
\text { SV/PP product; TPG: transpulmonary pressure gradient (mPAP-PAWP); WU: Wood units. Each p<0.05 } \\
\text { except where indicated NS (not significant) or NA (not applicable). }\end{array}$} \\
\hline
\end{tabular}




\begin{tabular}{|c|c|c|}
\hline & Ipc-PH & Cpc-PH \\
\hline Patients & 51 & 61 \\
\hline Age years & $69(64-76)$ & $72(63-79)$ NS \\
\hline Female sex & $31(61)$ & 41 (67) NS \\
\hline Body surface area $\mathrm{m}^{2}$ & $1.95(1.84-2.04)$ & $1.90(1.74-2.07) \mathrm{NS}$ \\
\hline Cardiac output L. $\min ^{-1}$ & $6.00(5.18-7.48)$ & $4.63(3.94-5.71)$ \\
\hline Cardiac index $\mathrm{L} \cdot \mathrm{min}^{-1} \cdot \mathrm{m}^{-2}$ & $3.15(2.74-3.73)$ & $2.44(2.14-2.92)$ \\
\hline Heart rate beats $\cdot \min ^{-1}$ & $73(62-85)$ & $80(64-92)$ NS \\
\hline Stroke volume $\mathrm{mL}$ & $86(68-108)$ & $61(48-86)$ \\
\hline Stroke volume index $\mathrm{mL} \cdot \mathrm{m}^{-2}$ & $45(36-54)$ & $31(26-40)$ \\
\hline mPAP $\mathrm{mmHg}$ & $34(29-37)$ & $46(37-52)$ \\
\hline Pulmonary arterial pulse pressure $\mathrm{mmHg}$ & $30(21-36)$ & $39(33-48)$ \\
\hline PAWP $\mathrm{mmHg}$ & $21(17-25)$ & $20(17-24)$ NS \\
\hline TPG mmHg & $11(9-16)$ & 23 (19-28) \\
\hline RAP $\mathrm{mmHg}$ & $12(10-15)$ & $14(11-20)$ \\
\hline PVR WU & $2.0(1.6-2.5)$ & $4.4(3.6-6.5) \mathrm{NA}$ \\
\hline PAC $\mathrm{mL} \cdot \mathrm{mmHg}^{-1}$ & $3.08(2.32-4.03)$ & $1.55(1.11-2.06)$ \\
\hline RC-time ms & $345(276-424)$ & $448(363-520)$ \\
\hline
\end{tabular}

Data are presented as $\mathrm{n}$, median (interquartile range) or $\mathrm{n}(\%)$. mPAP: mean pulmonary artery pressure. PAC: total pulmonary arterial compliance. PAH: pulmonary arterial hypertension. PAWP: pulmonary artery wedge pressure. PH: pulmonary hypertension. PVR: pulmonary vascular resistance. RAP: right atrial pressure. RC-time: the PVR times SV/PP product. TPG: transpulmonary pressure gradient (mPAP PAWPI. WU: Wood units. Each $p<0.05$ except where indicated NS (not significant) or NA (not applicable).

\section{Major dispersion of the PVR $\times P A C$ product (RC-time)}

The main assumption underlying the comparison of PAC at a given PVR level (RC-time constancy) was not verified. In both $\mathrm{Pc}-\mathrm{PH}$ and $\mathrm{PAH}$, the RC-time demonstrated extremely wide limits of agreement ( $95 \%$ confidence interval $= \pm 62 \%$ and $\pm 55 \%$, respectively) (table 3 and figure 2 ). In both groups, the dispersion of RC-time was higher than that of mPAP.

\section{Inconsistency of comparing PAC at a given PVR level concerning the total arterial load put on the} right ventricle

We first compared PAC at a given level of PVR. In the $62.6 \%$ patients in whom the comparison was feasible, PAC values at a given PVR level tended to be shifted downwards in $\mathrm{Cpc}-\mathrm{PH}$ as compared to iPAH, with a certain amount of overlap (figure 1 left panel). The tendency towards lower PAC at a given
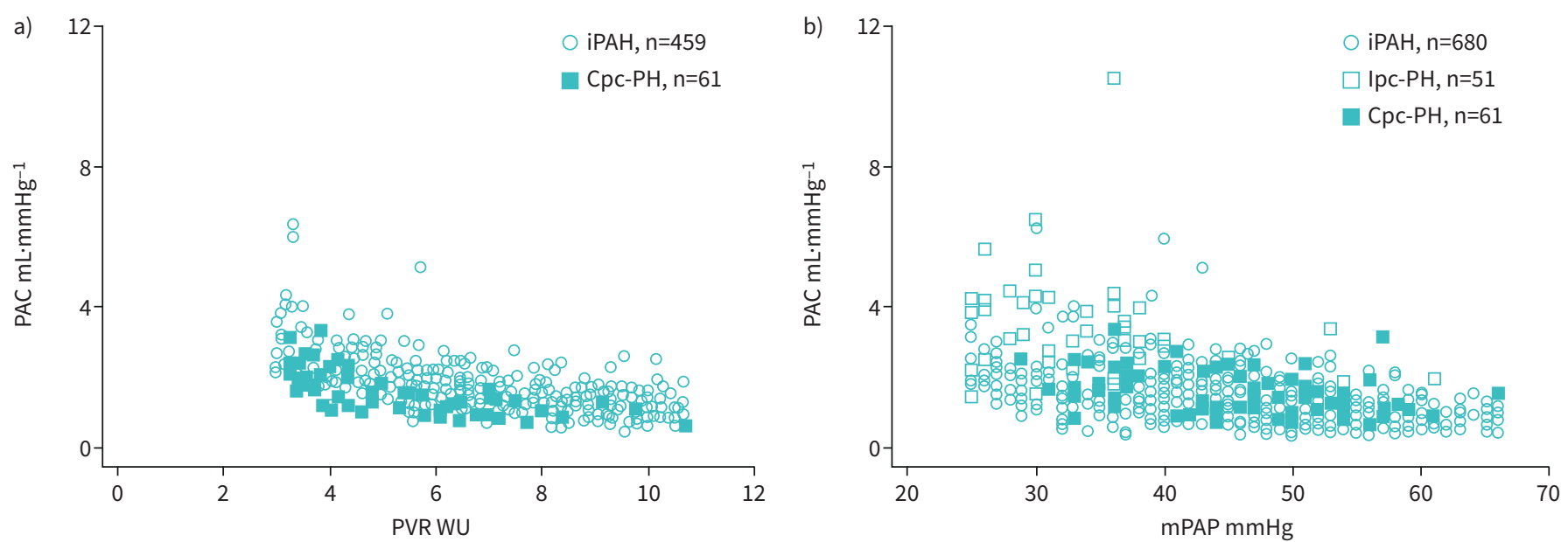

FIGURE 1 Scatter diagram showing the total pulmonary arterial compliance (PAC) plotted against overlapping pulmonary vascular resistance (PVR) (left panel) or overlapping mean pulmonary artery pressure (right panel). iPAH: idiopathic pulmonary arterial hypertension; Cpc-PH: combined pre- and postcapillary pulmonary hypertension; Ipc-PH: isolated postcapillary pulmonary hypertension. 


\begin{tabular}{|c|c|c|c|}
\hline Variable & QCD & IQR & $95 \%$ limits of agreement \\
\hline \multicolumn{4}{|l|}{$P c-P H(n=112)$} \\
\hline Body surface area & 0.07 & 0.14 & $\pm 24 \%$ \\
\hline Age & 0.10 & 0.21 & $\pm 32 \%$ \\
\hline mPAP & 0.18 & 0.39 & $\pm 48 \%$ \\
\hline RC-time & 0.21 & 0.42 & $\pm 62 \%$ \\
\hline \multicolumn{4}{|l|}{ iPAH (n=719) } \\
\hline Body surface area & 0.09 & 0.18 & $\pm 25 \%$ \\
\hline Age & 0.19 & 0.36 & $\pm 60 \%$ \\
\hline mPAP & 0.16 & 0.33 & $\pm 50 \%$ \\
\hline RC-time & 0.17 & 0.34 & $\pm 55 \%$ \\
\hline \multicolumn{4}{|c|}{$\begin{array}{l}\text { The data dispersion was quantified using the quartile coefficient of dispersion (QCD), the interquartile ratio } \\
\text { (IQR), and the acceptance range for } 95 \% \text { of the values. IQR }=(Q 3-Q 1) / \text { median. } Q C D=(Q 3-Q 1) /(Q 3+Q 1) \text {. Pc- } \\
\text { PH: postcapillary pulmonary hypertension; iPAH: idiopathic pulmonary hypertension; mPAP: mean } \\
\text { pulmonary artery pressure. RC-time: PVR } \times C \text { product. The } 95 \% \text { limits of agreement lacceptance range for } \\
95 \% \text { of the values) correspond to median } \pm \% \text { error. We considered a claim for the presence of a } \\
\text { "near-constant" variable in the database to be at least reasonable if the } 95 \% \text { limits of agreement fall } \\
\text { within the clinically acceptable range of up to } \pm 30 \% \text {, i.e., if } 95 \% \text { of the computed values were located } \\
\text { within } 30 \% \text { of the median value, lacceptance range from } 0.7 \times \text { median to } 1.3 \times \text { median). }\end{array}$} \\
\hline
\end{tabular}

level of PVR implies that total arterial tended to be lower in iPAH versus Cpc-PH. We also studied PVR values over the same PAC range. Overall, 788 (94.8\%) out of 831 patients entered the comparison, namely 111 out of $112 \mathrm{Pc}-\mathrm{PH}$ patients (99.1\%) and $677 \mathrm{iPAH}$ (94.2\%), with PAC ranging from 0.62-6.5 $\mathrm{mL} \cdot \mathrm{mmHg}^{-1}$. As compared to Ipc-PH, the PVR was, by definition, higher in iPAH studied at similar PAC level ranging from 1.14 to $6.5 \mathrm{~mL} \cdot \mathrm{mmHg}^{-1}$. When $\mathrm{Cpc}-\mathrm{PH}$ and $\mathrm{iPAH}$ patients were compared over the same PAC range $\left(0.62\right.$ to $\left.3.31 \mathrm{~mL} \cdot \mathrm{mmHg}^{-1}\right)$, PVR and thus total arterial load also
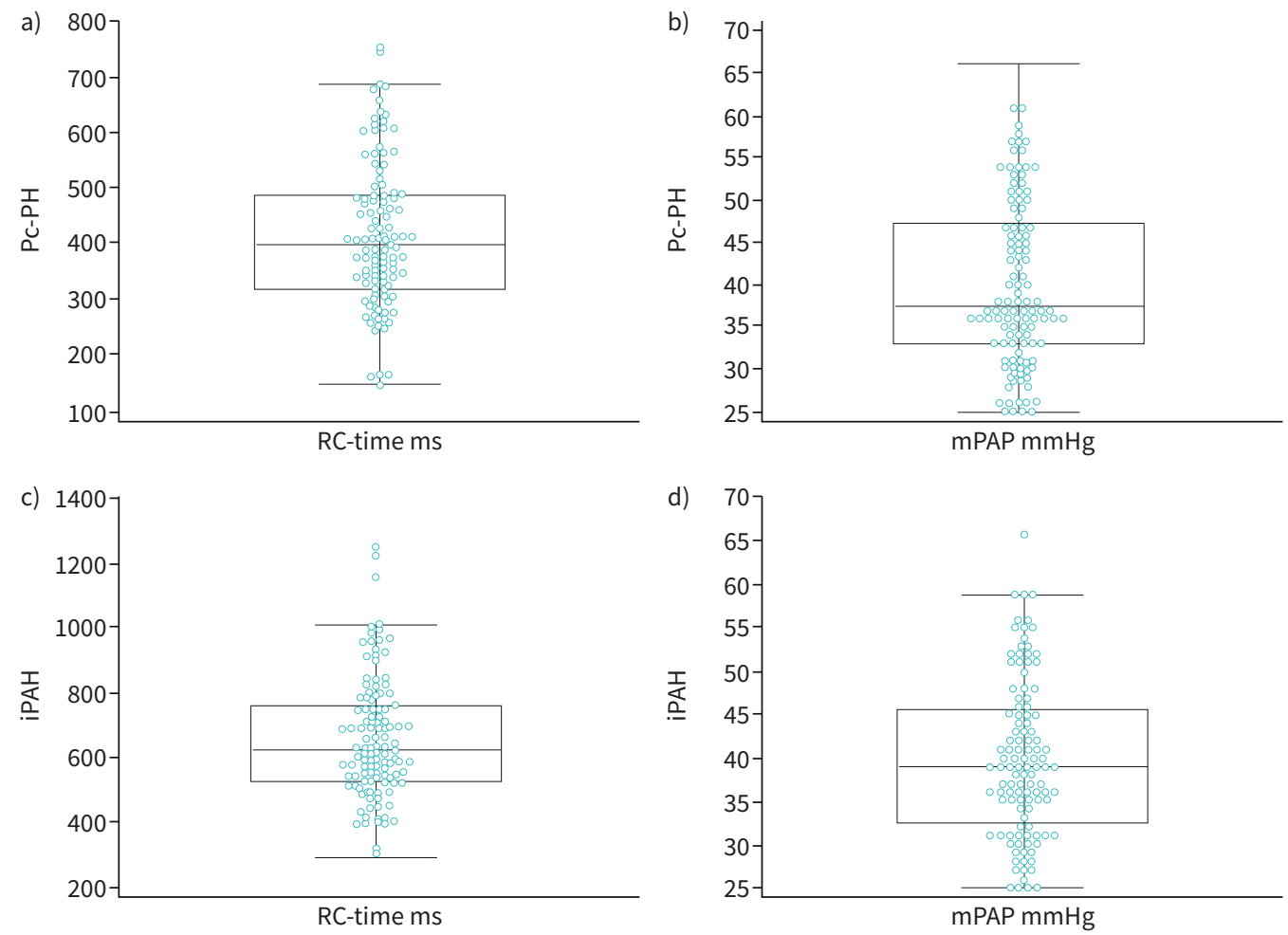

FIGURE 2 Box-and-whisker plots showing major dispersion of the pulmonary vascular resistance times total arterial compliance product (RC-time) and of mean pulmonary arterial pressure (mPAP) in postcapilliary pulmonary hypertension ( $\mathrm{Pc}-\mathrm{PH}$ ) and idiopathic pulmonary arterial hypertension (iPAH). 


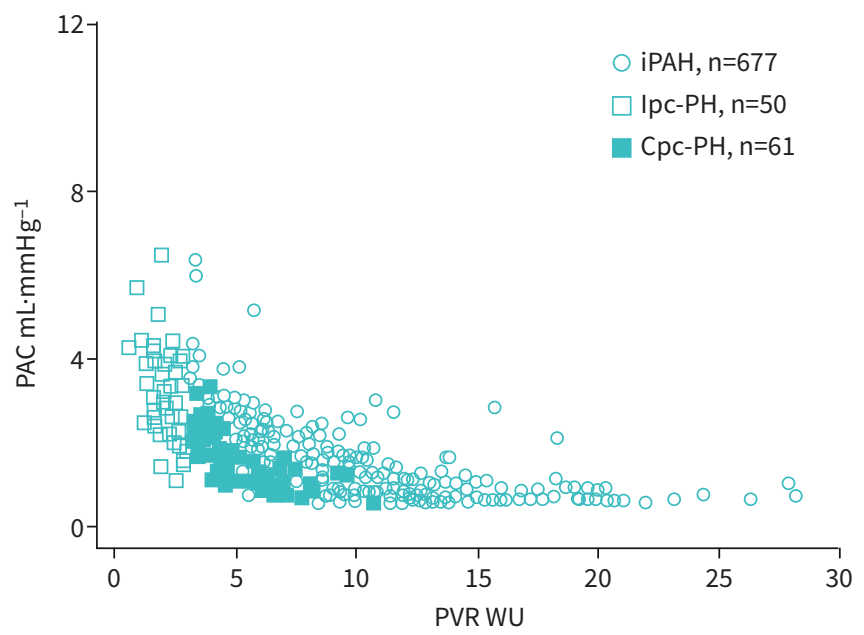

FIGURE 3 Scatter diagram showing pulmonary vascular resistance (PVR) plotted against overlapping total pulmonary arterial compliance (PAC). Open circles: patients with idiopathic pulmonary arterial hypertension (iPAH). Open squares: patients with isolated postcapillary pulmonary hyper-tension (Ipc-PH). Closed squares: patients with combined post-capillary and precapillary pulmonary hypertension ( $\mathrm{Cpc}-\mathrm{PH}$ ). Note that PVR is rightwards shifted in iPAH patients as compared to Pc-PH patients studied at a similar level of PAC.

tended to be higher in iPAH (figure 3). Finally 43 (5.2\%) out of 831 patients did not enter the comparison as their PAC was $<0.62 \mathrm{~mL} \cdot \mathrm{mmHg}^{-1}$ (in $42(5.8 \%$ ) out of $719 \mathrm{iPAH}$ ) or was extremely high $\left(10.5 \mathrm{~mL} \cdot \mathrm{mmHg}^{-1}\right.$ in 1 out of $\left.112 \mathrm{Pc}-\mathrm{PH}\right)$.

PAC values studied over the same mPAP range

Isobaric PAC tended to be slightly upwards shifted in Ipc-PH versus $\mathrm{iPAH}$ patients studied over the same 25-61 mPAP range, while iPAH and Cpc-PH demonstrated major overlap in their isobaric PAC over the same 29-66 mmHg mPAP range (figure $1 \mathrm{~b}$ ).

\section{Discussion}

Pulmonary hypertension is associated with stiffening of pulmonary arteries and enhanced pulsatile arterial load put on the right heart. To compare PAC (1/pulmonary arterial stiffness) between postcapillary and precapillary pulmonary hypertension, the chosen method must ideally allow comparison according to a strong physiological rationale and in the vast majority of the patients. The first result of our study was that one must clearly favour comparing PAC at fixed mPAP level (isobaric PAC) rather than at fixed PVR level for the following reasons: 1) there is a strong physiological rationale for comparisons based on isobaric PAC; and 2) mPAP values overlap between postcapillary and precapillary pulmonary hypertension in $95.3 \%$ of the study population, while PVR values overlap in $62.6 \%$ only. This may unify the way in which arterial compliance is studied in the pulmonary vascular bed and that used in all other vascular beds. The second result of our study was that from a fundamental physiological perspective, comparing PAC at each level of mPAP suggests that PAC is not lower in $\mathrm{Pc}-\mathrm{PH}$ as compared to iPAH. High left atrial filling pressure causes apparent stiffening of the pulmonary arteries by passive increases in mPAP. In Pc-PH patients, elevated left atrial pressure by itself is not associated with further intrinsic stiffening of the pulmonary artery compared with iPAH studied at similar mPAP level. Overall, this may lead to a reappraisal of the effects of high PAWP on the pulsatile and total arterial load put on the right heart.

In vascular beds of the body, arterial compliance is considered as mainly determined by the mean distending pressure, due to non-linear stress-strain behaviour of arteries. One exception is the pulmonary vascular bed, where it is widely admitted that PAC depends on vascular resistance (PVR) and downstream pressure (PAWP) [20-24]. The present study points to several inconsistencies in this approach. First, the comparison of PAC at a fixed PVR level was only feasible in less than two-thirds of the study population. By definition the comparison excluded the Ipc-PH patients, and it was feasible in only $63.2 \%$ iPAH. This lack of universal applicability appears to be a major limitation. Second, the classic belief that PAC may be accurately predicted by PVR alone in either group [20-24] was not verified. The RC-time was not constant but, on the contrary, highly variable and, in fact, more variable than MPAP in both groups. To continue to support the RC-time constancy or near-constancy would be as unrealistic as supporting that mPAP is constant or near-constant in pulmonary hypertension states. We have previously discussed [7, 30] the reasons why earlier studies have supported the opposite. Third, although it was confirmed that PAC 
studied at a given PVR level tended to be lower in Cpc-PH versus $\mathrm{PAH}$, there was a major overlap in PVR/PAC individual values. This was probably due to the natural heterogeneity of the various haemodynamic phenotypes encountered in pulmonary hypertension [12-19] resulting in an extremely variable RC-time. Fourth, concerning the total arterial load put on the right heart, no clear conclusion could be reached from the analysis of the PVR versus PAC relationships. Indeed, when patients were compared at a given PVR level (520 (62.6\%) out of 831), it was concluded that total arterial load tended to be lower in iPAH than Cpc-PH, while when patients were compared at a given PAC level (788 (94.8\%) out of 831), it was concluded that total arterial load tended to be higher in $\mathrm{PAH}$ than in both Ipc-PH and $\mathrm{Cpc}-\mathrm{PH}$. In an attempt to explain this aporia, the following points must be stressed: i) there are numerous limitations of the classic approach relying on the PVR versus PAC relationship, as detailed above; ii) it is likely that the contributions of PVR and PAC to total arterial load are overlapping rather than additive as they share SV and PA pressures in their formalism, thus leading to a major, problematic mathematical coupling [30]; and iii) the concept of total arterial load, which lumps the influences of PVR and PAC, seems questionable in our opinion, because for a given right ventricular geometry and in the absence of outflow tract obstruction, it is PAP which loads the right heart, not PVR nor PAC.

Comparing PAC values at fixed mPAP level (isobaric PAC) has strong physiological rationale and was feasible in the vast majority of the patients, namely all $\mathrm{Pc}-\mathrm{PH}$ and $94.6 \% \mathrm{iPAH}$. In our opinion, this is clearly a major advantage over the other method. Due to non-linear stress-strain behaviour of arteries [5, $6,25,26]$, arterial stiffness has to be measured under isobaric conditions to document potential intrinsic (i.e. pressure-independent) properties of the arterial wall. To quote the international recommendations in the field: "Be aware of the pressure dependence of arterial stiffness. If blood pressure differs between populations being studied, arterial stiffness has to be measured under isobaric conditions [26]". As mPAP increases, there is greater recruitment of relatively inelastic collagen fibres and, consequently, an increase in stiffness/a reduction in elasticity of the pulmonary arterial wall [5, 6, 31-33]. The two other reasons explaining the increased pulmonary arterial stiffness in pulmonary hypertension are the chronic stiffening due to wall thickening and vascular remodelling of the extracellular matrix, with loss of elastin and increase in collagen content; and stiffening due to smooth muscle cell responses [5, 6, 34]. Impedance studies have shown that there was a direct correlation between vessel stiffness and mPAP [4]. As far as the inverse relationship between PVR and PAC is concerned, MiLnor et al. [4] concluded that the main mechanism by which increased PVR would alter pulmonary arterial stiffness is by raising mPAP. Our study is consistent with milder intrinsic changes/remodelling of the arterial wall in Ipc- $\mathrm{PH}$ than in iPAH patients, while the same limits of compliance may well have been reached in Cpc-PH versus iPAH, and this point deserves further studies.

In $\mathrm{PAH}$, major abnormalities have been reported at the vascular tissue and cellular level and may contribute to pulmonary arterial stiffening $[35,36]$. In $\mathrm{Pc}-\mathrm{PH}$ patients, it is likely that the physiological explanation is that high left atrial pressure (which occurs as a consequence of systolic or diastolic dysfunction of the left ventricle and/or left atria) leads to higher mPAP by passive backward transmission of pressure. This results in lower PAC because of higher prevailing mPAP. With sustained elevation of pulmonary venous pressure, alterations in pulmonary vasoreactivity and structural damage at the arteriolar level ensue, responsible for a superimposed precapillary pulmonary hypertension component $(\mathrm{Cpc}-\mathrm{PH})[2$, $16,18,34]$. Our results are consistent the classic notion that elevated left heart filling pressures directly increase mPAP thus necessarily reducing PAC; and secondarily increase PVR through acute vasoconstriction and chronic vascular remodelling, which may further decrease PAC [16, 18]. In our study, iPAH patients with similar mPAP as $\mathrm{Cpc}-\mathrm{PH}$ patients exhibited a slightly lower PAC while PVR was markedly higher. Impedance studies performed in pulmonary hypertension have shown that for a given elevation of distending pressure, the stiffening of larger arteries in the patients with high resistance is greater than in the patients with normal resistance [4].

The Pc-PH patients had a mean RAP of $13 \mathrm{mmHg}$ suggesting that many of them were decompensated at the time point of right heart catheterisation. However, PAC was not associated with RAP, thus suggesting that the potential beneficial effects of decongestive therapy (e.g. diuretics), if observed, would be rather related to the treatment-induced decreases in mPAP.

The limitations of our retrospective study must be discussed. Pulmonary arterial load is at best assessed by the arterial input impedance in the frequency domain, but this is a complex approach, and as most clinical studies in this field, we used a time-domain approach. The new definition of pulmonary hypertension based on mPAP $>20 \mathrm{mmHg}$ [37] will not change our results as mPAP values in the 21-24 $\mathrm{mmHg}$ range will still overlap between pre and postcapillary pulmonary hypertension. Although a PVR threshold of 2.2 WU may be more clinically relevant for risk stratification [19], PVR $\geqslant 3$ WU remains the standard for defining pulmonary hypertension patients with a precapillary component. Finally, given the more than six-fold size difference between the two groups, we did not perform matching procedures. Although a clear-cut conclusion emerges, 
the study results were mainly qualitative, and further studies are needed to refine comparisons depending on the patient's hemodynamic phenotypes in the two pulmonary hypertension states.

The implications of our study should be discussed. Bearing in mind that the right ventricular function is a leading outcome determinant in patients with pulmonary hypertension, our study challenges the classic notion that PAC is lower in postcapillary pulmonary hypertension leading to more compromised right ventricular function than in precapillary pulmonary hypertension. The great variability of the PVR $\times \mathrm{SV} / \mathrm{PP}$ product (RC-time) implies that PVR and PA stiffness were differently affected by the disease process and thus may be potentially modified separately by therapeutic interventions. The analysis of isobaric PAC may help unify the way arterial compliance is studied in all vascular beds including the pulmonary circulation.

In conclusion, our study favours comparing PAC at fixed mPAP level (isobaric PAC) rather than at fixed PVR level. This may unify the way arterial compliance is studied in all vascular beds including that of the lungs. In Pc-PH, elevated left atrial pressure by itself was not associated with further intrinsic stiffening of the PA as compared to iPAH. A reappraisal of the effects of high PAWP on the pulsatile and total load put on the right heart is needed, and this point deserves further studies.

Acknowledgement: Part of the results of this study were presented at the ERS International Congress 2018, 15-19 September, Paris, France, and consequently appear as an abstract in the European Respiratory Journal [38].

Ethics statement: This retrospective study complied with the Declaration of Helsinki. Although French law does not require ethics committee approval or informed consent for retrospective data collection, the data collected were anonymised and complied with the requirements of the Commission Nationale Informatique et Liberté. This organisation dedicated to privacy, information technology and civil rights in France, approved the methods used to collect and analyse data (24 May 2003, approval number 842063).

Conflict of interest: D. Chemla has nothing to disclose. E. Berthelot has nothing to disclose. J. Weatherald reports grants, personal fees and nonfinancial support from Janssen Inc. and Actelion, personal fees and nonfinancial support from Bayer, and personal fees from Novartis, outside the submitted work. E.M.T. Lau has nothing to disclose. L. Savale reports grants, personal fees and nonfinancial support from Actelion, personal fees and nonfinancial support from MSD and Bayer, and grants, personal fees and nonfinancial support from GSK, outside the submitted work. A. Beurnier has nothing to disclose. D. Montani reports grants and personal fees from Actelion and Bayer, personal fees from GSK, personal fees from Pfizer, grants, personal fees and nonfinancial support from MSD, personal fees from Chiesi and Boehringer, and nonfinancial support from Acceleron, outside the submitted work. O. Sitbon reports grants, personal fees and nonfinancial support from Actelion Pharmaceuticals, personal fees and nonfinancial support from Bayer, grants from GlaxoSmithKline, grants, personal fees and nonfinancial support from Merck, and personal fees from Ferrer, Gossamer Bio and Acceleron, outside the submitted work. P. Attal has nothing to disclose. D. Boulate has nothing to disclose. P. Assayag has nothing to disclose. M. Humbert reports personal fees from Acceleron, grants and personal fees from Actelion and Bayer, and personal fees from GSK, Merck, Novartis, AstraZeneca and Sanofi, outside the submitted work. P. Hervé has nothing to disclose.

\section{References}

1 Humbert M, Guignabert C, Bonnet S, et al. Pathology and pathobiology of pulmonary hypertension: state of the art and research perspectives. Eur Respir J 2019; 53: 1801887.

2 Vachiery JL, Tedford RJ, Rosenkranz S, et al. Pulmonary hypertension due to left heart disease. Eur Respir J 2019; 53: 1801897.

3 Naeije R. Physiology of the pulmonary circulation and the right heart. Curr Hypertens Rep 2013; 15: 623-631.

4 Milnor WR, Conti CR, Lewis KB, et al. Pulmonary arterial pulse wave velocity and impedance in man. Circ Res 1969; 25: 637-649.

5 Lammers S, Scott D, Hunter K, et al. Mechanics and function of the pulmonary vasculature: implications for pulmonary vascular disease and right ventricular function. Compr Physiol 2012; 2: 295-319.

6 Schäfer M, Myers C, Brown RD, et al. Pulmonary arterial stiffness: toward a new paradigm in pulmonary arterial hypertension pathophysiology and assessment. Curr Hypertens Rep 2016; 18: 4.

7 Chemla D, Weatherald JC, Lau E, et al. Clinical and hemodynamic correlates of pulmonary arterial stiffness in incident, untreated patients with idiopathic pulmonary arterial hypertension. Chest 2018; 154: 882-892.

8 Lau EMT, Iyer N, Bailey BP, et al. Abnormal pulmonary artery stiffness in pulmonary arterial hypertension: In vivo study with intravascular ultrasound. PLoS ONE 2012; 7: e33331.

9 Sanz J, Kariisa M, Dellegrottaglie S, et al. Evaluation of pulmonary artery stiffness in pulmonary hypertension with cardiac magnetic resonance. JACC Cardiovasc Imaging 2009; 2: 286-295.

10 Stevens GR, Garcia-Alvarez A, Sahni S, et al. RV dysfunction in pulmonary hypertension is independently related to pulmonary artery stiffness. JACC Cardiovasc Imaging 2012; 5: 378-387.

11 Thenappan T, Prins KW, Pritzker MR, et al. The critical role of pulmonary arterial compliance in pulmonary hypertension. Ann Am Thorac Soc 2016; 13: 276-284.

12 Mahapatra S, Nishimura RA, Sorajja P, et al. Relationship of pulmonary arterial capacitance and mortality in idiopathic pulmonary arterial hypertension. J Am Coll Cardiol 2006; 47: 799-803.

13 Dupont M, Mullens W, Skouri HN, et al. Prognostic role of pulmonary arterial capacitance in advanced heart failure. Circ Heart Fail 2012; 5: 778-785.

14 Miller WL, Grill DE, Borlaug BA. Clinical features, hemodynamics, and outcomes of pulmonary hypertension due to chronic heart failure with reduced ejection fraction: pulmonary hypertension and heart failure. JACC Heart Fail 2013; 1: 290-299. 
15 Pellegrini P, Rossi A, Pasotti M, et al. Prognostic relevance of pulmonary arterial compliance in patients with chronic heart failure. Chest 2014; 145: 1064-1070.

16 Dragu R, Rispler S, Habib M, et al. Pulmonary arterial capacitance in patients with heart failure and reactive pulmonary hypertension. Eur J Heart Fail 2015; 17: 74-80.

17 Al-Naamani N, Preston IR, Paulus JK, et al. Pulmonary arterial capacitance is an important predictor of mortality in heart failure with preserved ejection fraction. JACC Heart Failure 2017; 3: 467-474.

18 Gerges M, Gerges C, Pistritto AM, et al. Pulmonary hypertension in heart failure. Epidemiology, right ventricular function, and survival. Am J Respir Crit Care Med 2015; 192: 1234-1246.

19 Maron BA, Brittan EL, Hess E, et al. Pulmonary vascular resistance and clinical outcomes in patients with pulmonary hypertension: a retrospective cohort study. Lancet Respir Med 2020; 8: 873-884.

20 Vonk Noordegraaf A, Westerhof BE, Westerhof N. The relationship between the right ventricle and its load in pulmonary hypertension. J Am Coll Cardiol 2017; 69: 236-243.

21 Lankhaar JW, Westerhof N, Faes TJ, et al. Quantification of right ventricular afterload in patients with and without pulmonary hypertension. Am J Physiol Heart Circ Physiol 2006; 29: H1731-H1737.

22 Lankhaar JW, Westerhof N, Faes TJ, et al. Pulmonary vascular resistance and compliance stay inversely related during treatment of pulmonary hypertension. Eur Heart J 2008; 29: 1688-1695.

23 Saouti N, Westerhof N, Postmus PE, et al. The arterial load in pulmonary hypertension. Eur Respir Rev 2010; 19: 197-203.

24 Tedford RJ, Hassoun PM, Mathai SC, et al. Pulmonary capillary wedge pressure augments right ventricular pulsatile loading. Circulation 2012; 125: 289-297.

25 Oliver JJ, Webb DJ. Noninvasive assessment of arterial stiffness and risk of atherosclerotic events. Arterioscler Thromb Vasc Biol 2003; 23: 554-566.

26 Van Bortel LM, Duprez D, Starmans-Kool MJ, et al. Clinical applications of arterial stiffness, Task Force III recommendations for user procedures. Am J Hypertens 2002; 15: 445-452.

27 Weatherald J, Boucly A, Chemla D, et al. The prognostic value of follow-up hemodynamic variables after initial management in pulmonary arterial hypertension. Circulation 2018; 137: 693-704.

28 Bonett DG. Confidence intervals for a coefficient of quartile variation. Comput Stat Data Anal 2006; 50: 2953-2957.

29 Critchley LA, Critchley JA. A meta-analysis of studies using bias and precision statistics to compare cardiac output measurement techniques. J Clin Monit Comput 1999; 15: 85-91.

30 Chemla D, Lau EM, Papelier Y, et al. Pulmonary vascular resistance and compliance relationship in pulmonary hypertension. Eur Respir J 2015; 46: 1178-1189.

31 Barman SA, Taylor AE. Effect of pulmonary venous pressure elevation on vascular resistance and compliance. Am J Physiol 1990; 27: H1164-H1170.

32 Zuckerman BD, Orton EC, Stenmark KR, et al. Alteration of the pulsatile load in the high-altitude calf model of pulmonary hypertension. J Appl Physiol 1991; 70: 859-868.

33 Newman JH, Brittain EL, Robbins IM, et al. Effect of acute arteriolar vasodilation on capacitance and resistance in pulmonary arterial hypertension. Chest 2015; 147: 1080-1085.

34 Guazzi M, Naeije R. Pulmonary hypertension in heart failure: pathophysiology, pathobiology, and emerging clinical perspectives. J Am Coll Cardiol 2017; 69: 1718-1734.

35 Ruffenach G, Chabot S, Tanguay VF, et al. Role for runt-related transcription factor 2 in proliferative and calcified vascular lesions in pulmonary arterial hypertension. Am J Respir Crit Care Med 2016; 194: 1273-1285.

36 Bertero T, Oldham WM, Cottrill KA, et al. Vascular stiffness mechanoactivates YAP/TAZ-dependent glutaminolysis to drive pulmonary hypertension. J Clin Invest 2016; 126: 3313-3335.

37 Simonneau G, Montani D, Celermajer DS, et al. Haemodynamic definitions and updated clinical classification of pulmonary hypertension. Eur Respir J 2019; 53: 1801913.

38 Chemla D, Berthelot E, Weatherald J, et al. Reappraising the effects of pulmonary artery wedge pressure on right ventricular pulsatile loading. Eur Respir J 2018; 52: Suppl. 62, PA3315. 